\title{
Optimization of Electroporation-Enhanced Intradermal Delivery of DNA Vaccine Using a Minimally Invasive Surface Device
}

\author{
Feng Lin,, Xuefei Shen,, Gleb Kichaev,, Janess M. Mendoza, Maria Yang, Philip Armendi,, Jian Yan, \\ Gary P. Kobinger, ${ }^{2}$ Alexander Bello, ${ }^{2}$ Amir S. Khan, ${ }^{1}$ Kate E. Broderick, and Niranjan Y. Sardesai'
}

\begin{abstract}
In vivo electroporation (EP) is an efficient nonviral method for enhancing DNA vaccine delivery and immunogenicity in animals and humans. Intradermal delivery of DNA vaccines is an attractive strategy because of the immunocompetence of skin tissue. We have previously reported a minimally invasive surface intradermal EP (SEP) device for delivery of prophylactic DNA vaccines. Robust antibody responses were induced after vaccine delivery via surface EP in several tested animal models. Here we further investigated the optimal EP parameters for efficient delivery of DNA vaccines, with a specific emphasis on eliciting cellular immunity in addition to robust humoral responses. In a mouse model, using applied voltages of 10-100 V, transgene expression of green fluorescent protein and luciferase reporter genes increased significantly when voltages as low as $10 \mathrm{~V}$ were used as compared with DNA injection only. Tissue damage to skin was undetectable when voltages of $20 \mathrm{~V}$ and less were applied. However, inflammation and bruising became apparent at voltages above $40 \mathrm{~V}$. Delivery of DNA vaccines encoding influenza virus $\mathrm{H} 5$ hemagglutinin (H5HA) and nucleoprotein (NP) of influenza H1N1 at applied voltages of 10-100 V elicited robust and sustained antibody responses. In addition, low-voltage (less than $20 \mathrm{~V}$ ) EP elicited higher and more sustained cellular immune responses when compared with the higher voltage (above $20 \mathrm{~V}$ ) EP groups after two immunizations. The data confirm that low-voltage EP, using the SEP device, is capable of efficient delivery of DNA vaccines into the skin, and establishes that these parameters are sufficient to elicit both robust and sustainable humoral as well as cellular immune responses without tissue damage. The SEP device, functioning within these parameters, may have important clinical applications for delivery of prophylactic DNA vaccines against diseases such as HIV infection, malaria, and tuberculosis that require both cellular and humoral immune responses for protection.
\end{abstract}

\section{Introduction}

D NA VACCINATION has been extensively explored in both animal models and the clinic and holds great promise as an effective molecular therapy for infectious diseases and cancers (Sardesai and Weiner, 2011). Central to the mechanism of DNA vaccination, the delivered plasmids express proteins that act as antigens that have the ability to elicit both humoral and cellular immune responses. In contrast, conventional vaccines induce mainly a biased helper $\mathrm{T}$ cell type 2 (Th2) immune response. Therefore, a DNA vaccine strategy is especially suitable for pathogens such as human immunodeficiency virus type 1 (HIV-1), malaria, and tuberculosis that require a strong cellular immune response for protection.
Injection of DNA vaccine into muscle or skin results in transfection of host cells, expression of antigen, and priming of immune responses directed toward the expressed antigen (Tang et al., 1992; Ulmer et al., 1993; Wang et al., 1993). Intracellular antigen synthesis gives rise to effective antigen presentation on major histocompatibility complex (MHC) class I and efficient priming of $\mathrm{CD}^{+}$cytotoxic $\mathrm{T}$ lymphocyte (CTL) responses, which might be helpful to eradicate pathogens that colonize inside cells (Corr et al., 1996; Fu et al., 1997; Iwasaki et al., 1997; Kutzler and Weiner, 2008). These unique features make DNA vaccines suitable not only for preventive but also therapeutic approaches against cancers and chronic infectious diseases.

Although DNA vaccination holds great promise, the platform has been limited through inability to efficiently

\footnotetext{
${ }^{1}$ Inovio Pharmaceuticals, Blue Bell, PA 19422.

${ }^{2}$ Special Pathogens, National Microbiology Laboratory, Public Health Agency of Canada, Winnipeg, Manitoba, Canada.
} 
deliver the plasmid intracellularly. Delivery of naked DNA into muscle or skin by standard injection results in weak immunogenicity, even at high DNA doses. Many techniques have been investigated to improve DNA delivery efficacy. Delivery methods include the use of viral and bacterial vectors, various chemical formulations such as liposomes, and physical delivery mechanisms such as ultrasound, lasers, gene gun, and electroporation (EP) (Bolhassani et al., 2011). Although viral vectors allow for excellent transfection efficiency, it comes with a risk of viral vector-associated severe inflammation when delivered at high doses (Lehrman, 1999; Ferber, 2001) and vector-induced immune responses resulting in a compromised boosting effect (Hirao et al., 2010b).

In vivo $\mathrm{EP}$ has been shown to be an efficient nonviral method for delivering DNA vaccines both preclinically and clinically (Luxembourg et al., 2007; Bodles-Brakhop and Draghia-Akli, 2008; Sardesai and Weiner, 2011). EP is able to enhance DNA delivery up to 1000-fold as compared with naked injection of the plasmid, primarily through enhanced intracellular uptake (Mir et al., 1998; Mathiesen, 1999). EP has been shown to enhance the immunogenicity of DNA vaccines in small and large mammals such as mice, guinea pigs, rabbits, and pigs (Widera et al., 2000; Ahlen et al., 2007; Draghia-Akli et al., 2008; Muthumani et al., 2008), and nonhuman primates (Babiuk et al., 2002; Otten et al., 2004; Luckay et al., 2007; Laddy et al., 2009; Hirao et al., 2010a). Data demonstrate that a 10- to 100-fold augmentation of immunity that results in protection from pathogenic challenge has been achieved in various animal models against diseases such as simian immunodeficiency virus (SIV) and HIV (Luckay et al., 2007; Hirao et al., 2008a; Rosati et al., 2009; Hirao et al., 2010b), human papillomavirus (HPV) (Best et al., 2009), hepatitis C virus (HCV) (Ahlen et al., 2007), influenza (Laddy et al., 2008, 2009), and malaria (Dobano et al., 2007).

EP-mediated delivery of DNA vaccine into muscle tissue has been extensively studied and shown to be highly effective. The success of the intramuscular EP platform may be due to the relatively large area of tissue transfected and the long-lasting expression of the transgene in muscle cells (Brown et al., 2008; Tjelle et al., 2008). However, more recently, EP-mediated delivery targeting the skin has been attracting considerable attention (Hirao et al., 2008b). The skin as an immunization target tissue has advantages over muscle and mucosa in that it is easily accessible and can be visibly monitored. Most importantly, it is a highly immune-reactive tissue due to the abundance of antigen-presenting cells (APCs) such as Langerhans cells in the epidermis, and dermal dendritic cells in the dermis (Babiuk et al., 2000; Toebak et al., 2009). Thus far, a number of experimental EP devices have been developed to target the skin.

Devices for intradermal EP can generally be classified into various categories depending on their modes of action. Contactless electrodes can consist of a static spark (Broderick et al., 2011a) and make no direct contact with the skin of patients. Examples of noninvasive or surface electrodes are devices such as caliper-type electrodes (Zhang et al., 1996) and the four-plate electrode (4PE; Heller et al., 2007) platform, which use plates as electrodes. The multielectrode array (MEA) (Heller et al., 2010; Donate et al., 2011) and meander electrodes which consist of an array of interweaving electrode fingers of alternating polarity (Zhang et al., 2002), are additional examples. In general, these platforms make direct contact with the skin surface without rupturing the stratum cornea of the skin, and require relatively high electrical field strength for efficient transfection. Another configuration, called the plate-and-forktype electrode, consists of a noninvasive plate and an invasive fork electrode (Maruyama et al., 2001). Low-voltage parameters were reported when using the plate-and-fork-type electrode for skin/subcutaneous delivery, however, the efficiency was weak, demonstrating only an approximate 2-fold increase when $12-24 \mathrm{~V}$ was used (Maruyama et al., 2001). The invasive device configurations consist of arrays of multiple needles that penetrate into the skin for EP-mediated DNA delivery. Roos and colleagues (2009) reported that a device consisting of two parallel rows of four-needle electrodes (eight in total) using two pulses of $1125 \mathrm{~V} / \mathrm{cm}$ and eight pulses of $275 \mathrm{~V} / \mathrm{cm}$ field strength resulted in high immune responses. However, a topical anesthetic cream was suggested for use to decrease the sensation of pulse delivery in patients.

We have most recently reported two skin EP devices: the CELLECTRA-3P and the surface EP (SEP). The CELLECTRA-3P is a minimally invasive device that targets dermal and subcutaneous layers of the skin (Hirao et al., 2008b) with mild EP conditions and minimal tissue damage. The device consists of three-needle ( $3 \mathrm{~mm}$ in length) electrodes forming a triangular microarray to cover the DNA injection site. Hirao and colleagues (2010a) reported that a multivalent smallpox DNA vaccine delivered by the CELLECTRA-3P device can elicit polyvalent immunity in macaques, and offers protection from a highly pathogenic monkeypox challenge. The second novel surface EP device is the minimally invasive SEP device (Broderick et al., 2011b). This device makes contact with the surface of the skin to overcome the stratum cornea layer and targets the epidermis of the skin. This device consists of a $4 \times 4$ electrode array at $1.5-\mathrm{mm}$ spacing to provide optimal coverage of the DNA injection site. In addition, this tight electrode spacing allows for the use of low applied voltages without sacrificing delivery efficiency. Using this SEP device to deliver DNA vaccines, high and persistent antibody immune responses can be induced in animal models (Broderick et al., 2011b). However, the optimal EP parameters for the generation of a sustained and balanced immune response remains to be further investigated. Specifically, there is impetus to elucidate the electrical field that is able to elicit not only a robust antibody immune response but also strong cellular immune responses, while at the same time preserving tissue integrity.

In this study, we assessed various electrical field strengths for EP, using the SEP device, to determine the optimal applied voltage for efficient and balanced DNA vaccine delivery into the skin with minimal tissue damage. In particular, we analyzed the effect of voltage on the induction of both antibody and cellular immune responses in a mouse model. The results may provide a guideline for desirable EP field strengths when assessing various disease targets, especially as these concepts move toward broad prophylactic DNA vaccine delivery in humans.

\section{Materials and Methods}

\section{Plasmids and synthetic linear expression cassettes}

Plasmids pGX2001 and pMB75.6-NP were prepared inhouse using an EndoFree kit (Qiagen, Valencia, CA). Plasmids gWiz-GFP and gWiz-Luc were purchased from 
Aldevron (Fargo, ND). pGX2001 is a SynCon vaccine construct (Inovio Pharmaceuticals, Blue Bell, PA) that encodes a consensus sequence of hemagglutinin of $\mathrm{H} 5$ influenza virus (H5HA). pMB75.6-NP vaccine encodes nucleoprotein (NP) of the Puerto Rico influenza H1N1 strain. Matched NP sequence from the Puerto Rico/39 strain was optimized, synthesized, and cloned into the backbone of the clinically approved mammalian expression vector, pMB75.6. The linear expression cassettes (LECs) used in this research were manufactured by Vandalia (Huntington, WV). Briefly, LEC pcrNP was originated from pMB75.6-NP, using PCR-based technology, and LEC pcrM2 was amplified from the pMB75.6-M2 construct, which encodes M2 of influenza A/ New Caledonia/99. The LEC contains elements essential for expression in mammalian cells: a cytomegalovirus (CMV) promoter, intron, and gene of interest followed by the simian virus 40 (SV40) polyadenylation signal. All plasmids and LECs were diluted in $1 \times$ phosphate-buffered saline (PBS) before injection.

\section{Animals}

Female BALB/c mice were purchased from Charles River Laboratories (Wilmington, MA) and used in transgene GFP and luciferase expression and immunization studies. Hartley guinea pigs, female, 4 weeks of age with weights between 300 and $350 \mathrm{~g}$, were also used in a histology study. All animals were housed and handled at Bio-Quant (San Diego, CA) in accordance with the standards of the Institutional Animal Care and Use Committee.

\section{GFP and luciferase expression and analyses}

Five groups of mice $(n=5)$ were anesthetized with isoflurane ( 5 and $3 \%$ for induction and maintenance, respectively). Sites on each flank were shaved and cleaned carefully. gWiz-GFP at $2.0 \mathrm{mg} / \mathrm{ml}$ and gWiz-Luc at $0.5 \mathrm{mg} / \mathrm{ml}$ were injected separately into the skin (intradermal, $30 \mu \mathrm{l}$ of each plasmid) followed by EP at 10, 20, 40, 60, and $100 \mathrm{~V}$. Control mice $(n=5)$ were injected with the same dose of plasmid DNA without EP. The mice were killed on day 4, and skin samples were taken by skin punches and frozen at $-20^{\circ} \mathrm{C}$. The GFP samples were lined up for image analyses, using OV-100 fluorescence microscope (Olympus America, Center Valley, PA). For the luciferase samples, skin biopsies were quick-frozen in liquid nitrogen and ground to a powder with a mortar and pestle. Samples were resuspended in $1 \times$ lysis buffer with further homogenization at room temperature. After a brief centrifugation, the supernatants were analyzed under standard assay conditions according to the protocol of the luciferase assay system (cat. no. E4030; Promega, Madison, WI).

\section{Histological examination of skin at EP sites}

Guinea pigs were injected intradermally with plasmid DNA (50 $\mu$ l per injection) followed without or with EP at different voltages. Guinea pigs were killed humanely on day 4 , and the skin samples were harvested by skin punch and fixed at $4 \%$ paraformaldehyde overnight for cryosectioning. Twenty-micrometer sections were cut, and the tissue sections were stained with a Histo-Perfect H\&E staining system (BBC Biochemical, Mount Vernon, WA) according to the manu- facturer's protocol. In H\&E staining, hematoxylin stains nuclei blue while the cytoplasm is stained pink with eosin. The presentation of the total area of blue nuclear staining is a crude method to determine inflammatory cell infiltration. An infiltration of inflammatory cells into skin results in an increase in the area of blue staining roughly proportional to the severity of the response.

\section{Immunization and electroporation in mice}

Mice were randomized into seven groups $(n=10)$. After 1 week of acclimation, animals were sedated with inhaled isoflurane, and sites on the flank were shaved properly for intradermal injection and electroporation. DNA vaccine pGX2001 or pMB75.6-NP (each, $10 \mu \mathrm{g}$ in $30 \mu \mathrm{g}$ PBS) was injected intradermally followed immediately by electroporation at various voltage settings (from 10 to $100 \mathrm{~V}$, three pulses with each pulse length $100 \mathrm{msec}$ ). A boost immunization was conducted at week 3 . Animals were bled retroorbitally at week 2 , week 4 (1 week after boost), and week 6 (3 weeks postboost) and serum from blood samples was harvested for ELISA. Three animals from each group were killed at each time points for splenocyte enzyme-linked immunospot (ELISPOT) assay.

\section{ELISA for detection of antibody end-point titers}

Antibody responses against $\mathrm{H} 5 \mathrm{HA}, \mathrm{NP}$, and M2e (the extracellular domain of the M2 protein) were evaluated by ELISA, using serum from immunized mice as previously described (Lin et al., 2011). Briefly, Costar 96-well EIA/RIA plates were coated with recombinant H5HA (cat. no. IT-0030051p, $0.3 \mu \mathrm{g} / \mathrm{ml}$; Immune Technology, New York, NY), NP of influenza A (cat. no. IMR-274, $1.0 \mu \mathrm{g} / \mathrm{ml}$; Imgenex, San Diego, CA), or M2e peptide (15 $\mu \mathrm{g} / \mathrm{ml}$; Invitrogen, Carlsbad, $\mathrm{CA})$ at $4^{\circ} \mathrm{C}$ overnight. After washing, the plates were blocked for nonspecific binding by adding $200 \mu \mathrm{l}$ of PBS with $0.5 \%$ bovine serum albumin (BSA) for $1 \mathrm{hr}$ at $37^{\circ} \mathrm{C}$. Serum samples were diluted at 1:50 in dilution buffer (PBS with 0.2\% BSA and $0.05 \%$ Tween 20) and then serial dilution (1:3) was performed in the plate from the first row for each sample. After incubation for $2 \mathrm{hr}$ at $37^{\circ} \mathrm{C}$ and subsequent washing, anti-mouse IgG-biotin (cat. no. B9904; Sigma-Aldrich, St. Louis, MO) was diluted 1:10,000, added to each well, and incubated for $1 \mathrm{hr}$ at $37^{\circ} \mathrm{C}$ before washing. This was followed by adding $50 \mu \mathrm{l}$ of streptavidin-horseradish peroxidase (HRP) (diluted 1:2000; Southern Biotech, Birmingham, AL) to each well followed by incubation for $1 \mathrm{hr}$ at $37^{\circ} \mathrm{C}$ before washing. The final step was done by adding $50 \mu \mathrm{l}$ of HRP substrate (P9187; Sigma-Aldrich) followed by incubation at room temperature in the dark for $10 \mathrm{~min}$ before reading the optical density (OD) at $450 \mathrm{~nm}$. A reading was considered positive if the $\mathrm{OD}$ was higher than the mean $+3 \times \mathrm{SD}$ from prebled serum, and the positive titer was plotted as end-point titer.

\section{IFN- $\gamma$ ELISPOT assay}

Two weeks after the first immunization, and 1 and 3 weeks after the second immunization, splenocytes from three mice of each group were isolated. Single-cell suspensions were cleared of red blood cells, using RBC lysis buffer (eBioscience, San Diego, CA). The ELISPOT assay kit was purchased from R\&D Systems (Minneapolis, MN). ELISPOT 
plates (96-well; Millipore, Billerica, MA) were coated with anti-mouse interferon (IFN)- $\gamma$ monoclonal antibody. After overnight incubation at $4^{\circ} \mathrm{C}$, the wells were washed and blocked with blocking buffer according to the manufacturer's protocol. Pooled splenocytes from each group were added to the wells and incubated overnight with a $10 \mu \mathrm{g} / \mathrm{ml}$ concentration of NP147 (TYQRTRALV) (Jeon et al., 2002; Lin et al., 2011), NP55 (RLIQNSLTIERMVLS) (Jeon et al., 2002), or M2e (MSLLTEVETPIRNEWGCRCNDSSD) (all from Invitrogen). The plates were then washed and developed according to the protocol described by the manufacturer. The spots were counted and analyzed by Cellular Technology (Shaker Heights, OH).

\section{LEC influenza immunization, using low- and high- voltage EP delivery and influenza virus challenge}

$\mathrm{BALB} / \mathrm{c}$ mice in groups of 10 were immunized with LEC influenza vaccines by surface EP device at weeks 0,3 , and 6, using low-voltage $(15 \mathrm{~V})$ and high-voltage $(30 \mathrm{~V})$ EP delivery. Five micrograms of LEC pcrNP and $3.8 \mu \mathrm{g}$ of LEC pcrM2 (mole equivalent) were used per mouse for each immunization. At week 8, blood samples were taken from each mouse to study antibody responses against antigens. Splenocytes were harvested and pooled from three mice in each group to study T cell responses against NP and M2e. Peptides NP147 and M2e-P10 (MSLLTEVETPIRNEWGCRCNDSSD) (Wolf et al., 2011) were used in the cellular immune response assay. At week 10, influenza challenge experiments were conducted according to Biosafety Level IV protocols at the National Microbiology Laboratory, Public Health Agency of Canada (Winnipeg, MB, Canada) according to ethics guidelines that had been approved by their ethics committee. A dose of H1N1 influenza strain A/Canada/AB/RV1532/2009 equal to $5 \times 10^{5}$ TCID (tissue culture infective dose)/mouse was used. Mice were monitored for survival and body weight every day for 21 days after challenge.

\section{Statistical analysis}

All values are presented as means $\pm S E$ unless otherwise stated. An unpaired Student $t$ test (two-tailed) was used when comparing two groups. $p<0.05$ was considered statistically significant. For the challenge study, a MannWhitney nonparametric statistical test was used to compare mouse body weight loss between different groups. All statistical analysis was performed with GraphPad Prism 4 for Windows (GraphPad Software, San Diego, CA).

\section{Results}

Reporter gene expression varies with EP voltage applied to skin, using surface EP device

We have previously reported on a minimally invasive dermal surface EP device for administering DNA vaccination in a prophylactic setting using low-voltage parameters (Broderick et al., 2011b). Although immunization with this device platform resulted in robust antibody responses, we were also interested in optimizing EP parameters for cellular immune responses and observing the combined kinetics of such responses. Initially, to assess the effect of varying voltage parameters on expression levels in skin, reporter plasmids encoding green fluorescent protein (GFP) and lu- ciferase (Luc) were delivered via intradermal injection combined with surface EP in a mouse model. Sixty micrograms of plasmid encoding GFP or $15 \mu \mathrm{g}$ of plasmid encoding Luc was injected separately into the skin of mice. Injection of plasmid was immediately followed with (or without) surface EP at applied voltages ranging from 10 to $100 \mathrm{~V}$. Animals were killed humanely on day 4 and the treated skin area was excised for either GFP expression analysis or processing for use in a Luc activity assay. Representative images of GFP expression in mouse skin are shown in Fig. 1A. GFP expression was detected in all EP groups. However, the most robust expression was observed in groups that received voltages between 10 and $60 \mathrm{~V}$. GFP expression in the group receiving the highest voltage $(100 \mathrm{~V})$ was visibly lower than was observed in all other EP groups. To quantify GFP expression, pixel counting was carried out after fluorescence microscopy, under standardized conditions (Fig. 1B). As seen in Fig. $1 \mathrm{~A}$ and $\mathrm{B}$, minimal or undetectable GFP expression was observed in mice that received GFP plasmid injection alone (no EP). As compared with the no-EP group, all EP groups showed significantly higher levels of GFP expression $(p<0.05)$. However, expression in the $100-\mathrm{V}$ group was significantly lower than in the 20-, 40-, and 60-V groups $(p<0.05)$. To confirm the GFP expression analysis, a quantifiable Luc assay was used to assess Luc expression in mouse skin. As shown in Fig. 1C, Luc expression was significantly increased when electroporation was applied compared with Luc plasmid injection only (no EP). Robust Luc activity was detected in all EP groups, which were significantly higher than the no-EP group $(p<0.05)$, with a trend toward higher activity at the higher voltages. Nevertheless, Luc activity in the 40-, 60-, and 100-V groups was significantly higher $(p<0.05)$ than in either the $10-$ or $20-\mathrm{V}$ group. Immediately after EP treatment (data not shown) and on sacrifice of the animals (Fig. 1D), we observed the direct effect of EP parameters on mouse skin. Using visual observation, we scored each animal for redness, swelling, and necrosis at the treatment site resulting from the application of $\mathrm{EP}$ at various voltages. For the EP groups that received applied voltages of $20 \mathrm{~V}$ or less, no visible tissue damage was observed. Notable tissue damage was observed at the treatment sites in all other groups with applied voltage greater than $20 \mathrm{~V}$. The skin damage observed in the $40 \mathrm{~V}$ group was relatively minor compared with higher voltages. Redness, swelling, and scabbing of skin was particularly pronounced in the 60 and $100 \mathrm{~V}$ groups both immediately after treatment and persisting until the point of sacrifice ( 3 days postdosing).

EP-related skin damage was further assessed in guinea pigs under the EP conditions described in the previous mouse study. Histological examination was performed for each treatment on skin from the treatment sites sampled 4 days after plasmid gWiz-GFP injection followed without or with EP at various voltages. Naive skin without any treatment was harvested for control. As shown in Fig. 2, as compared with naive control, plasmid injection without EP (no EP) (Fig. 2B) caused a mild inflammatory response, an approximately 1.5-fold increase in infiltration as assessed by the dark blue nuclear staining, which consisted mainly of macrophages and neutrophils. Plasmid injection followed by $\mathrm{EP}$ at $20 \mathrm{~V}$ showed a similar increase in infiltration as the non-EP control. However, EP at the higher voltages-60 and 
A
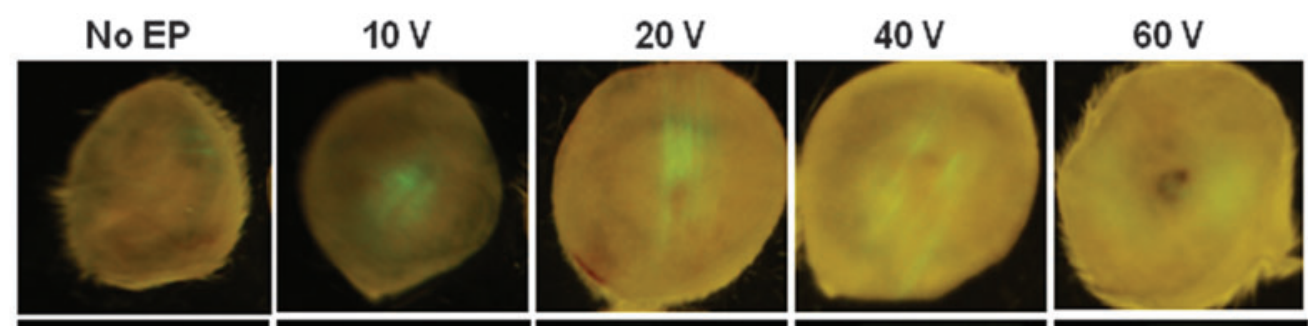

$100 \mathrm{~V}$
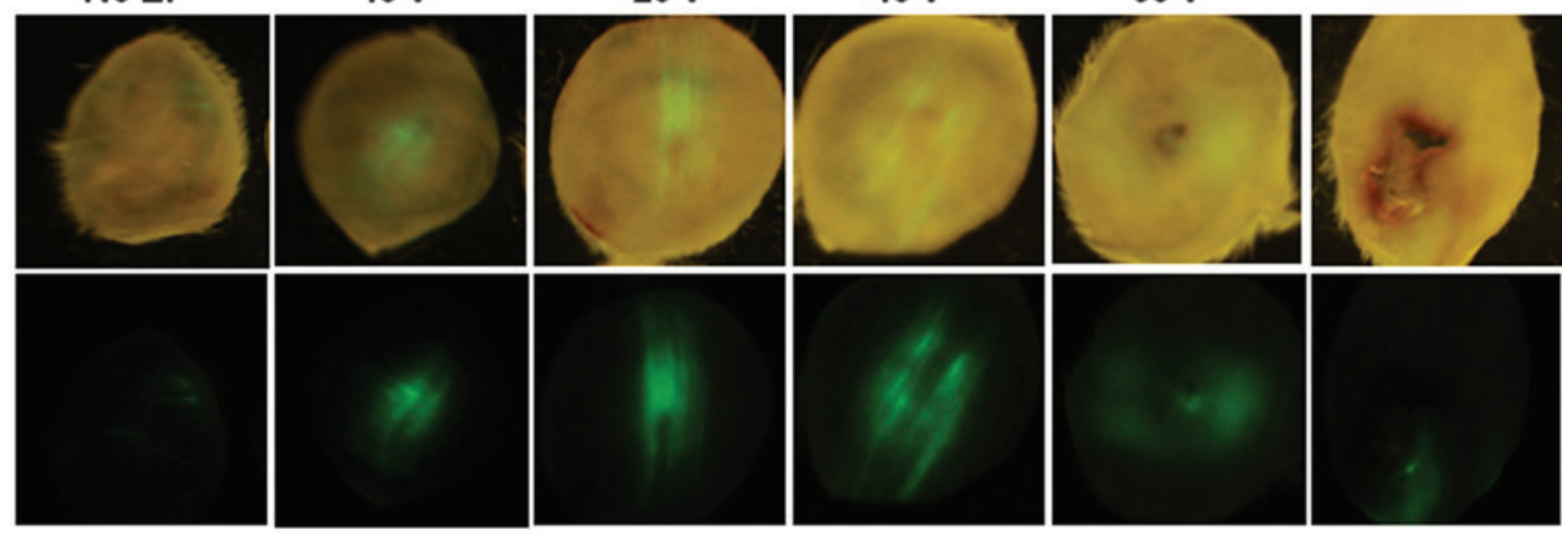

B

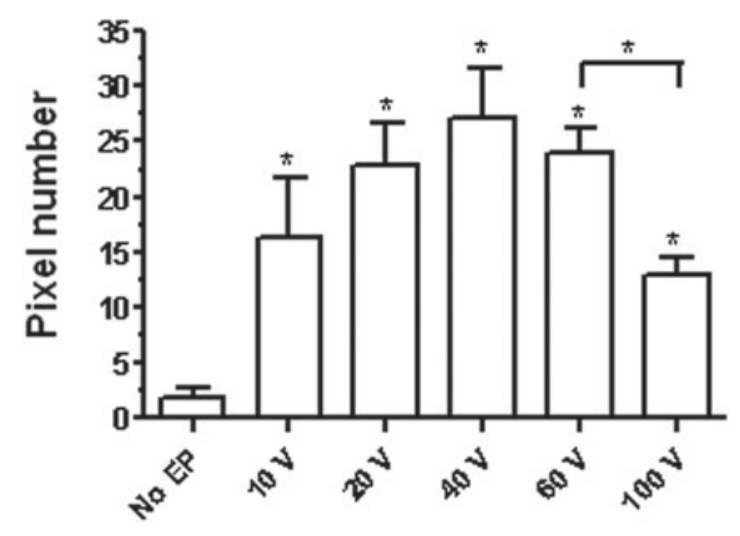

D
C

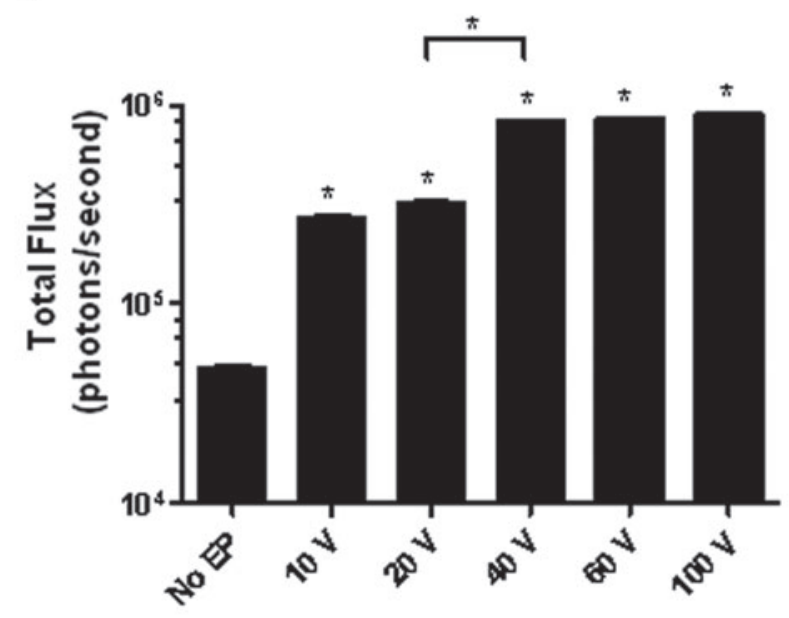

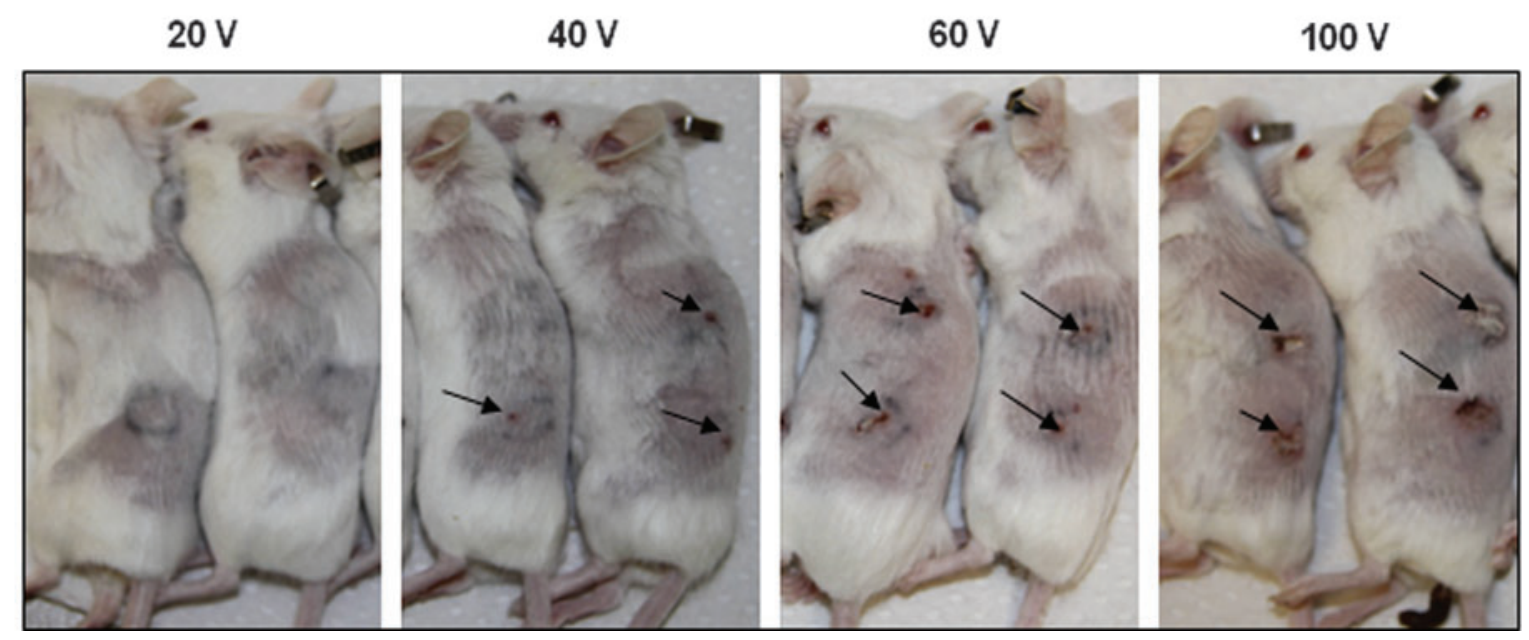

FIG. 1. Expression in mouse skin of transgenes encoding GFP and luciferase, delivered by a surface intradermal electroporation (SEP) device at various voltages. Plasmids gWiz-GFP and gWiz-Luc were injected separately into the skin of five mice in each group, followed by EP. Expression levels of GFP and Luc were analyzed 4 days after plasmid DNA delivery, using the SEP device at various voltages. (A) GFP expression in skin. The top row of images were taken under both visible and fluorescent light, and the bottom row was taken under fluorescent light only. (B) GFP expression intensity, determined by pixel counting. The pixel number for each group is shown as the mean and SE. (C) Luc activity in skin. The Luc activity in each group is shown as the mean and SE. (D) Skin damage (arrows) caused by various EP voltages. ${ }^{*} p<0.05$ (Student $t$ test). 

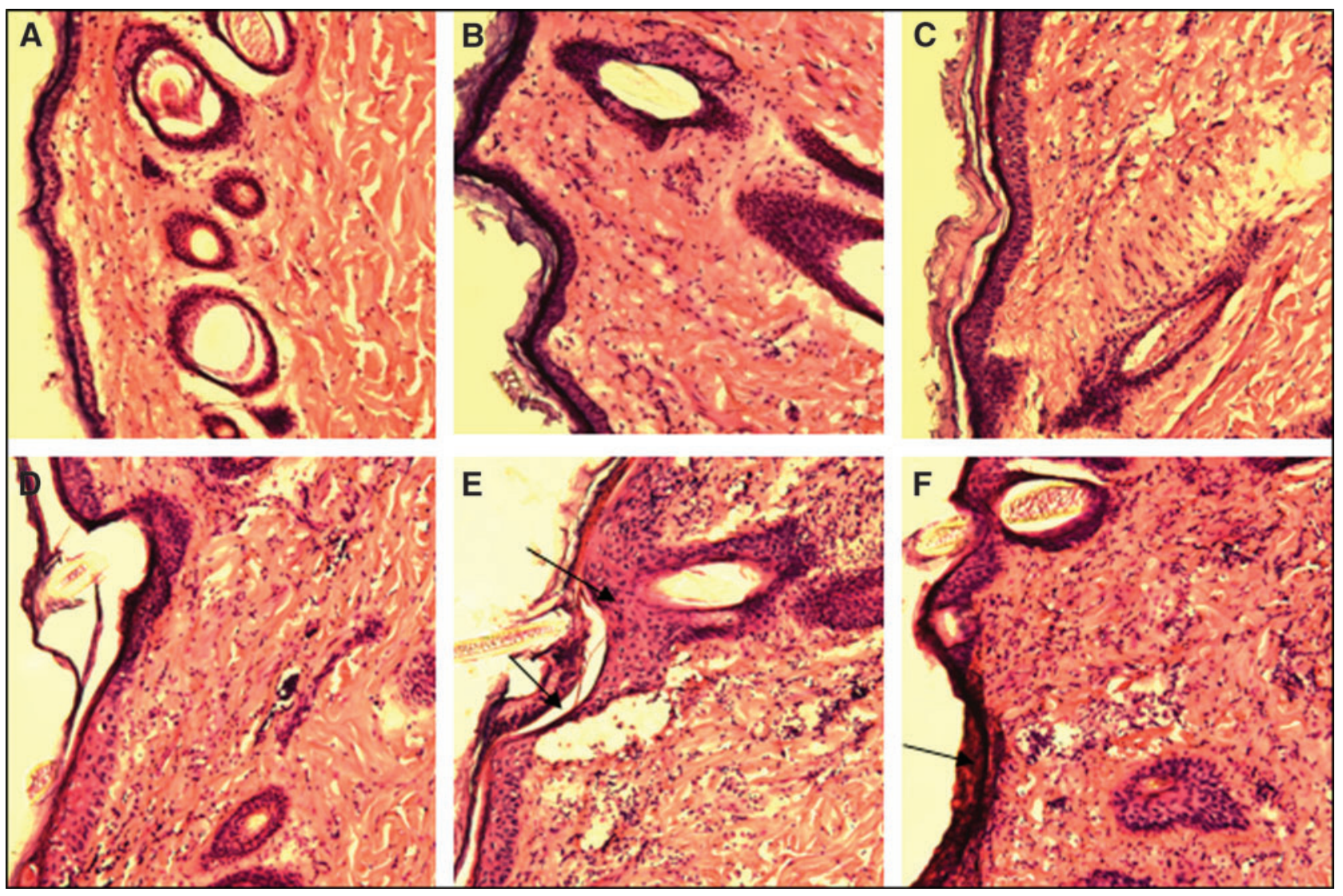

FIG. 2. H\&E staining of skin 4 days after vaccination at various EP voltages. All microscopy images were taken in visible light at an original magnification of $\times 10$. (A) Naive skin without any treatment; (B) skin injected with plasmid without EP (no EP); (C-F) skin injected with plasmid followed by EP at 20, 40, 60, and $100 \mathrm{~V}$, respectively. Arrows indicate the epidermal damage likely caused by electrode EP.

$100 \mathrm{~V}$ (Fig. 2E and F)—caused necrosis in the epidermal layer where the needle/electrode array made direct contact with the skin, in addition to substantially greater inflammation (4- to 6-fold increase in leukocyte influx). EP at $40 \mathrm{~V}$ (Fig. 2D) demonstrated only moderate inflammation, with an approximate 3 -fold increase in leukocyte infiltration.

\section{A range of EP voltages results in robust humoral immune responses}

EP-enhanced DNA vaccine delivery by surface EP in skin is known to augment immune responses against the encoded antigen in various species (Broderick et al., 2011b). To elucidate the association between immune response and EP applied voltage, we evaluated humoral immunity in mice after DNA immunization with the surface EP device, using voltages ranging from 5 to $100 \mathrm{~V}$. A schematic of the immunization protocol is detailed in Fig. 3A. Animals were vaccinated on day 0 and boosted at week 3 with a uniform dose of DNA plasmid encoding a consensus sequence of the hemagglutinin antigen (HA) from influenza A/H5N1 (H5HA) and nucleoprotein (NP) of influenza A/H1N1 (Puerto Rico) either with or without EP. Antibody responses at weeks 2, 4, and 6 were analyzed by ELISA. As shown in Fig. 3B, the antibody end-point titers in all EP groups were significantly higher than that in the control group (DNA injection only [no EP]) after the second immunization. The average end-point titer was 8- to 20-fold higher than in the control group. The antibody titers in mice treated with EP at $5 \mathrm{~V}$ initially showed no difference relative to the control group at the week 2 time point, yet increased to the same level as other EP groups at the week 4 and 6 time points. The same trend in antibody responses was observed on immunization with NP plasmid (Fig. 3C).

\section{Lower voltage EP parameters result in stronger cellular immune responses}

The responses detailed in Fig. 3 demonstrate that robust antibody titers can be induced when DNA plasmids are delivered into skin via EP, using parameters as low as $5 \mathrm{~V}$. To assess the effect of voltage parameters on the cell-mediated immune response, we further analyzed and compared the ability of various EP voltages to elicit NP-specific $\mathrm{CD}^{+} \mathrm{T}$ cell responses. Splenocytes from naive and vaccinated groups of mice were incubated with or without the MHC class I $\left(\mathrm{H}-2 \mathrm{D}^{\mathrm{b}}\right)$-restricted NP147 peptide or NP55 (helper T epitope peptide) to assess antigen-specific $\mathrm{CD}^{+}$or $\mathrm{CD}^{+} \mathrm{T}$ cell responses. As shown in Fig. 4A, 2 weeks postpriming, mice in the control group (injected with DNA only) showed 128 spots and the group electroporated at $5 \mathrm{~V}$ had 168 spots. However, at this time point, groups that received EP at $10 \mathrm{~V}$ and above had significantly more spots than the $5 \mathrm{~V}$ group or no-EP control group. The group that received EP at $100 \mathrm{~V}$ 

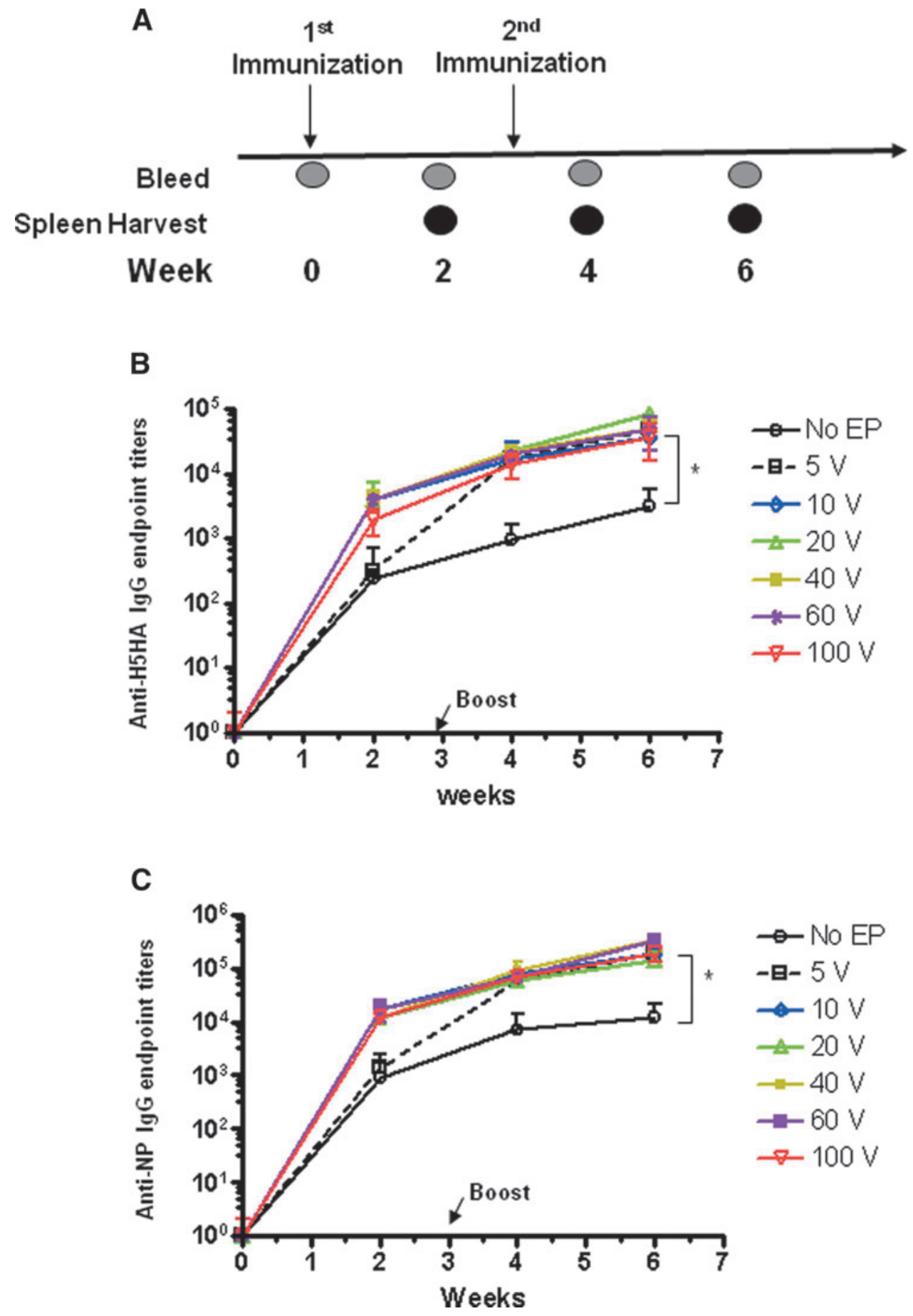

FIG. 3. Anti-H5HA and anti-NP antibody responses in mice. Plasmids encoding H5HA and NP sequences were delivered by SEP delivery at various voltages. (A) Experimental design. (B) Anti-H5HA and (C) anti-NP antibody end-point titers. Mice $(n=10)$ were immunized with pGX2001 and pMB75.6-NP on day 0 and boosted at week 3. Blood samples were harvested at weeks 2, 4, and 6 for antigen-specific antibody titer analysis by ELISA. The end-point titers are shown as geometric means and SE. ${ }^{*} p<0.05$ (all EP groups compared with no-EP group at week 4 and 6 by Student $t$ test).

had the highest number of spots (656), significantly higher than in the $60 \mathrm{~V}$ group $(p<0.05)$. In Fig. $4 \mathrm{~B}$, at week $4(1$ week postboost), the spots in response to both type of peptides showed trends similar to those observed at week 2 after priming. In response to NP147, all EP groups above $10 \mathrm{~V}$ elicited significantly increased spot numbers relative to the no-EP control group. In terms of the responses to NP55, all of the EP groups demonstrated significantly increased spot numbers compared with the no-EP control. However, at this time point, the highest number of spots was detected in the 10V group, with 1122 spots in response to NP147 and 1262 spots in response to NP55, whereas the $100 \mathrm{~V}$ group had a lower number of spots compared with the $10 \mathrm{~V}$ to $60 \mathrm{~V}$ groups. At week 6 (4 weeks after boost), the profile of spot 

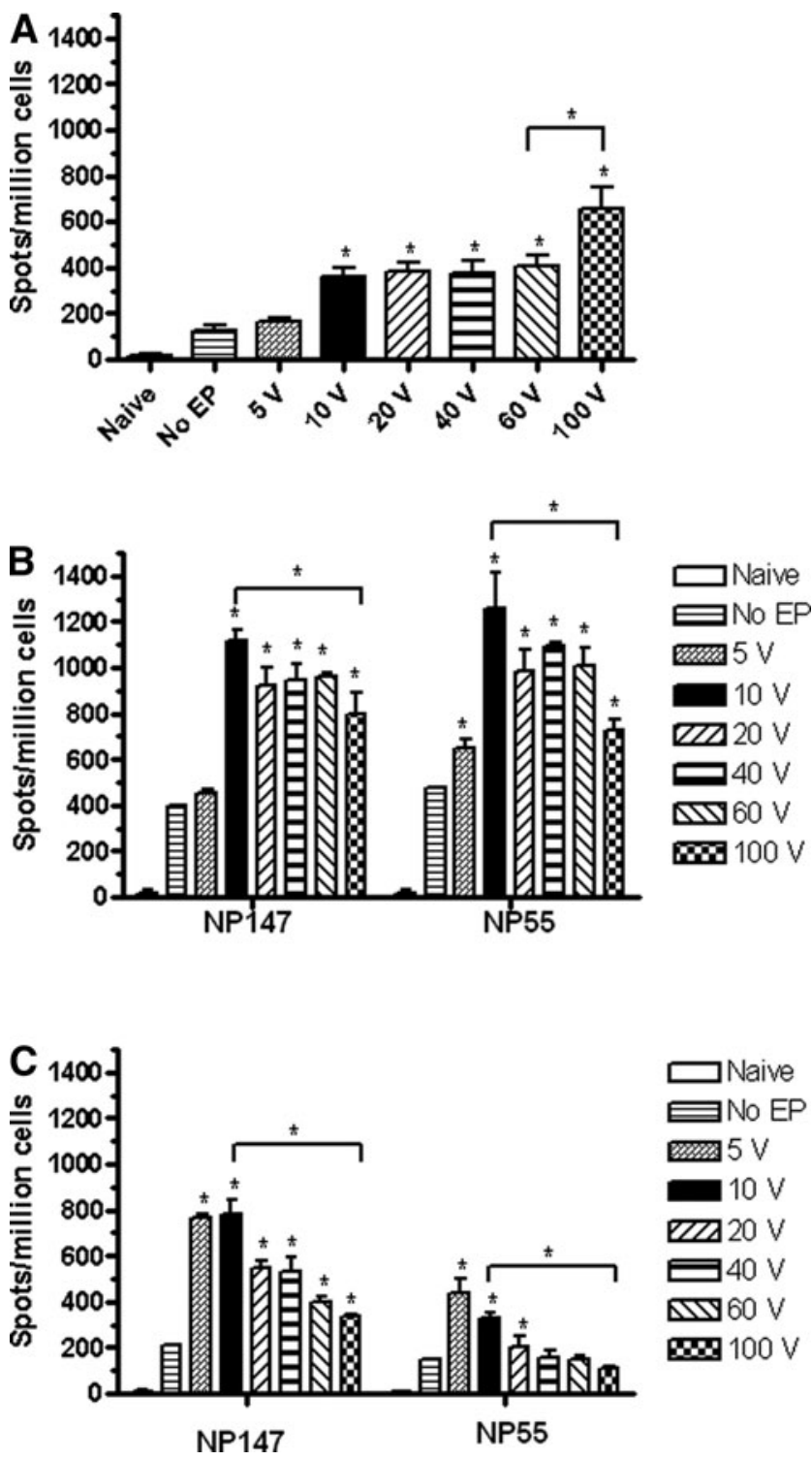

FIG. 4. Cellular immune response to NP antigen after prime at week 0 and boost at week 3 with pMB75.6-NP immunization at various EP voltages. (A) IFN- $\gamma$ ELISPOTs at week 2 after priming. Splenocytes were incubated overnight with or without NP CTL epitope peptide NP147. (B and C) IFN- $\gamma$ ELISPOTs at, respectively, weeks 4 and 6 (a boost at week 3). Splenocytes were incubated overnight with or without both CTL peptide NP147 and helper T epitope peptide NP55. Samples were run in triplicate in these assays. Responses to NP147 are shown as spot number (means and SE). ${ }^{*} p<0.05$.

numbers dropped significantly for all groups except the group receiving $\mathrm{EP}$ at $5 \mathrm{~V}$; their responses actually increased compared with 1 week postboost (Fig. 4C). Compared with the response to the NP147 peptide, splenocytes from all groups in response to NP55 peptide showed lower overall spot numbers, yet the general trend remained similar.

\section{Low- and high-voltage delivery of LECs and protection from challenge}

An alternative DNA vaccination strategy to plasmid DNA is the use of linear expression cassettes (LECs). These pieces of DNA are generated through PCR technology and contain only the coding region of a gene. As detailed in the preceding sections, we have observed that DNA vaccination delivered by EP, using parameters less than $20 \mathrm{~V}$, induced both robust antibody and cellular immune responses in mice. Although the generation of robust immune responses is impressive, the real test of a vaccination platform is its ability to protect animals in a disease challenge model. In this challenge experiment, we assessed whether EP voltage parameters would affect the ability of an influenza DNA vaccine to protect mice against a nonlethal disease challenge. Here we further compared the ability of the SEP device, using lowand high-voltage parameters, to deliver LEC influenza vaccines. BALB/c mice (10 per group) were immunized with equal moles of an LEC expressing influenza NP (pcrNP) and an LEC expressing influenza M2 (pcrM2). Both DNA vaccines were injected intradermally either with or without EP at low voltage $(\mathrm{LV}, 15 \mathrm{~V})$ or high voltage $(\mathrm{HV}, 30 \mathrm{~V})$, using the SEP device. Mice were bled 2 weeks after the last boost; antibody and cellular responses against NP and M2 were measured by ELISA and ELISPOT, respectively. As shown in Fig. 5A, strong and consistent antibody responses were induced with both the LV and HV parameters. Antibody titers were significantly higher than with intradermal delivery of the DNA vaccine alone (no EP). CTL and helper T lymphocyte (HTL) responses with both parameter sets are shown in Fig. 5B. Vaccination with EP (both LV and HV parameters) generated significantly higher numbers of NP CTL and M2e HTL epitope-specific spots as compared with DNA injection only (no EP). However, mice in the LV group generated 1.6fold higher NP CTL spots than did the HV EP group. There was no difference in M2e HTL spot numbers between the HV and LV parameter groups. In an attempt to assess whether the responses induced by LV and HV EP were protective, mice were then challenged with the current human/swine Flu strain A/Canada/AB/RV1532/2009 at $5 \times 10^{5}$ TCID/ mouse 10 weeks after the last immunization. Because this strain is nonlethal in a mouse model, the body weights of the mice were monitored as a readout of morbidity. As shown in Fig. 5C, all groups of mice lost weight initially. On the basis of a Mann-Whitney nonparametric statistical test, there was no significant difference between the various groups. However, we did see a trend toward mice vaccinated with LECs via EP appearing to recover faster than the naive and injection without EP (no-EP) groups. Indeed, the difference was statistically significant on days 8 and 9 postchallenge, based on a Student $t$ test.

\section{Discussion}

In the current study, we have demonstrated that efficient, balanced, and sustained immune responses can be elicited by DNA vaccination, using skin EP delivery at low electrical field strengths with the SEP device. Using parameters between 5 and $10 \mathrm{~V}$, DNA vaccines delivered by the SEP device can induce both high antibody responses and robust, sustained cellular immune responses in comparison with higher voltage parameters in mice. Low-voltage parameters were also used to deliver LECs (another format of DNA vaccine) in mice and resulted in robust immune responses. At these lower voltages, no tissue damage was visualized and we believe that application of such voltages would be highly 

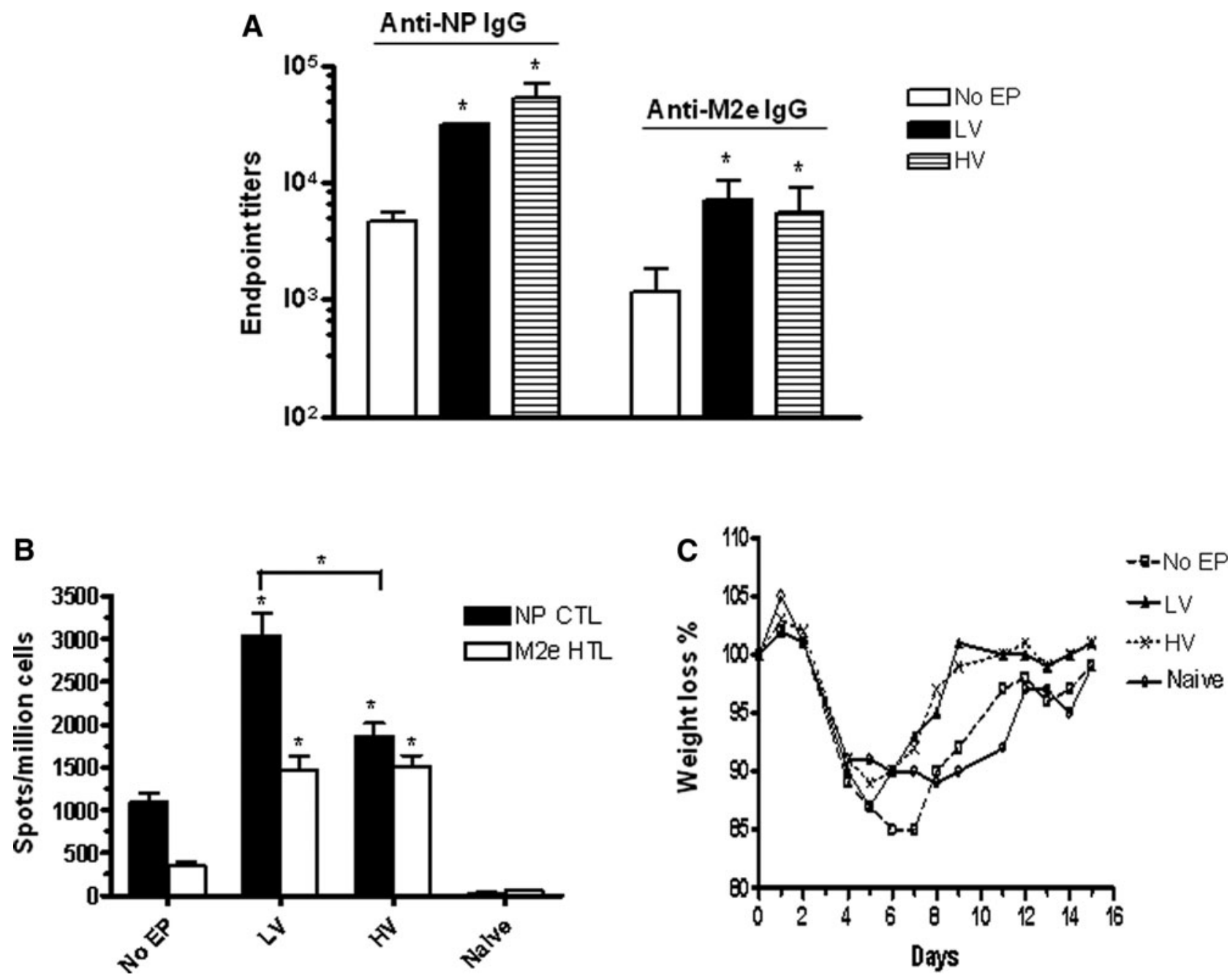

FIG. 5. Low- and high-voltage delivery of linear expression cassette (LEC) and influenza virus challenge. Mice were immunized with LEC DNAs in the presence or absence of EP at low voltage $(15 \mathrm{~V})$ and high voltage $(30 \mathrm{~V})$ at weeks 0,3 , and 6. At week 8, blood samples were collected for analysis of antibody and cellular responses. At week 10, mice were challenged with H1N1 influenza strain A/Canada/AB/RV1532/2009, at $5 \times 10^{5}$ TCID/mouse. Mice were monitored for survival and body weight every day for 21 days after challenge. (A) Anti-NP and anti-M2e IgG antibody responses. (B) IFN- $\gamma$ ELISPOTs. (C) Mouse body weight loss after challenge. ${ }^{*} p<0.05$.

tolerable. These optimized parameters may have excellent tolerance from a patient's perspective while maintaining vaccination efficiency. Therefore, such a device and parameter combination could be used for broad prophylactic DNA vaccine intradermal delivery in humans, including children.

We have previously shown that reporter genes can be successfully delivered into skin in various animal models such as guinea pig, rabbit, swine, and mouse by using the surface device at low-voltage EP (Broderick et al., 2011b). However, the effect of EP electrical field strength on transfection efficiency, immune response (both humoral and cellular), and tissue damage remained to be further investigated. We initially assessed influence of a range of voltages from 10 to $100 \mathrm{~V}$ on reporter transgene expression level and tissue damage in mouse skin, using the surface device. Transgene expression of GFP and Luc with this mode of EP begins to be visible $6 \mathrm{hr}$ after treatment, reaches its peak between days 3 and 5, and persists for more than 2 weeks (unpublished data; Inovio Pharmaceuticals). We chose day 4 as the time point of sacrifice and harvest for this study because it fell in the peak expression range. GFP expression was found mainly on the underside of mouse skin. This pattern differs from other animal models previously tested in that expression is generally confined to the upper layers of the epidermis. We have previously shown that, using this EP platform in a guinea pig model, the majority of GFP transfection occurred in the stratum granulosum cell layer of the epidermis; a small number of GFP-positive cells was also detected in the stratum basale (Broderick et al., 2011b). From these data, we can hypothesize that the majority of transfected cells would be keratinocytes, because $90 \%$ of the epidermis consists of these cells. This alteration in expression localization between guinea pig and mouse is due primarily to the thin nature of mouse skin. Optimal transgene expression of GFP was seen in between the applied voltages of $10-40 \mathrm{~V}$. The GFP signal was less robust in the $60 \mathrm{~V}$ group and significantly impacted in the $100 \mathrm{~V}$ group. We believe this is a direct result of quenching of the GFP signal by 
hemoglobin, because both the excitation and emission spectra of GFP overlap with peaks in the absorption spectra of hemoglobin (Lane and Smith, 1999). The presence of hemoglobin is a result of tissue damage caused by the high-voltage parameters. Tissue damage/necrosis was observed in all applied voltages greater than $40 \mathrm{~V}$, and became severe as the voltage increased to $100 \mathrm{~V}$. The damage was concomitant with moderate to severe inflammation in the treatment sites. Tissue damage results from localized burning of the skin caused by the high electrical field strength $(>300 \mathrm{~V} / \mathrm{cm})$. These parameters are often used with other intradermal EP devices such as plate electrodes (Zhang et al., 1996) and invasive needle electrodes (e.g., eight-needle array; Roos et al., 2009). This may explain the recommendation for use of a topical anesthetic cream to decrease the sensation of pulse delivery in patients. In parallel to the GFP localization, we also conducted a luciferase expression study. As we observed with the GFP study, all EP parameters significantly enhanced Luc expression in skin. However, in contrast to the GFP study, the highest Luc activity was observed in the higher voltage groups $(40-100 \mathrm{~V})$. This observation may further reflect the effect of GFP quenching by hemoglobin in the damaged tissue, because hemoglobin should have no inhibitory effects on Luc activity.

We next determined whether vaccination performed with EP at various voltages in the skin elicited different antibody and cellular immune responses. After the first immunization, humoral immune responses in mice treated with EP at $10 \mathrm{~V}$ or above produced significantly higher titers than did the $5 \mathrm{~V}$ group or no-EP group. There is an absolute requirement at these ultralow voltages $(5 \mathrm{~V})$ for excellent electrical contact. If such contact is not achieved, we believe that the transfection can be directly impacted. This may explain the lack of antibody response at the first time point for the $5 \mathrm{~V}$ group. However, after boost, the antibody titers from the $5 \mathrm{~V}$ group were as high as those of all the other EP groups, and all were significantly higher than that in the control group. This finding confirms that EP enhances the immune response relative to naked DNA injection alone but also demonstrates that there is no added benefit of voltages greater than $10 \mathrm{~V}$ to deliver DNA into the skin, because of the potential tissue damage as revealed in Fig. 1D.

In the cellular immune response assay, we used two different epitope peptides to conduct IFN- $\gamma$ ELISPOTs: CTL epitope peptide NP147 and helper T epitope NP55. In response to NP147 at the first time point (week 2 after the first vaccination), increasing voltage trended toward higher spot numbers. Mice in the $100 \mathrm{~V}$ group demonstrated the highest spot numbers (656). This may be attributed to the adjuvant effect of tissue damage at this voltage. At this time point, no significant difference was observed between the $5 \mathrm{~V}$ group and the no-EP group. These results demonstrate that EP at $10 \mathrm{~V}$ or more gave rise to a robust cellular immune response after only a single immunization. At week 4 (or 1 week after boost), the spot number in the $10 \mathrm{~V}$ group was the highest in response to both CTL (1100) and helper T (1250) epitope peptides compared with all other EP groups. However, the levels across the 10 to $100 \mathrm{~V}$ range did not vary widely. At week 6 (or 3 weeks after boost), the trend in spot numbers changed. The lowest voltage EP groups (5 and $10 \mathrm{~V}$ ) showed the highest spot numbers whereas, interestingly, the spot numbers significantly decreased in all the higher voltage groups (Fig. 4C). These findings indicate that DNA vaccination delivered into skin via our SEP device may elicit a robust cellular immune response at voltages as low as $5 \mathrm{~V}$. In terms of the kinetics of the cellular immune response, it appears that higher EP voltages elicited faster T cell responses, but that these are not sustained over time and are not improved with boosting. In this study, we observed that the $100 \mathrm{~V}$ group elicited the highest CTL response after the first immunization; however, the response decreased quickly after the boost and continued to decrease over time. In contrast to this, the low EP voltage groups ( 5 and $10 \mathrm{~V}$ ) elicited slower $\mathrm{T}$ cell responses but were boostable and resulted in a more sustained response that continued to increase over the time period we assessed. Although the exact cause of these response magnitudes and kinetics is not clear, it is possible that the high levels of antigen expression combined with the adjuvant effect of tissue damage in the high-voltage groups might have maximized the response, resulting in an inability to boost further. If similar cellular response trends observed here in mice were to be maintained in humans, these results might suggest that, for therapeutic or pandemic vaccination, it would be prudent to initially use a higher EP voltage parameter for priming, as this could elicit faster $\mathrm{T}$ cell responses. For prophylactic vaccination purposes, however, the lower EP voltage might be the optimal choice for both prime and boost, to produce a more sustained response. For therapeutic applications, combining a high-voltage prime with a lower voltage boost may produce an optimal high and fast initial response with more sustained $\mathrm{T}$ cell kinetics over time, but this would need further experimental clarification in other animal models.

In previous work (Broderick et al., 2011b; Lin et al., 2011) we compared the SEP modality with standard intramuscular EP delivery. The results demonstrated that low-voltage intradermal EP with the SEP device gave rise to comparable, if not in some cases superior, immune responses as compared with intramuscular EP.

To establish the effects of voltage on immune correlates of protection, we performed a challenge study in mice using two voltage parameter sets, low $(15 \mathrm{~V})$ and high $(30 \mathrm{~V})$ voltages, to deliver a linear expression cassette (LEC) as a DNA vaccine. In this experiment, we found that delivery of LECs with low EP voltage could induce comparable antibody responses to the high-voltage parameters and indeed even more robust cellular immune responses than in the high-voltage group. This suggests that multiple nucleotide formats, that is, plasmid and LEC, are enhanced by lowvoltage SEP delivery. The elicited protection in challenge appears to be correlated with the resulting immune responses. This finding suggests that surface EP DNA vaccination at the highly tolerable low voltage is able to induce robust immune responses for protection against virus challenge, although there was no significant difference between the various groups, based on a Mann-Whitney nonparametric statistical test. However, a trend toward mice vaccinated with LECs via EP appearing to recover faster than naive mice was observed. In a separate study, we performed a lethal mouse challenge study using the low-voltage parameter of the SEP device. In the study, using low-voltage parameters, we were able to demonstrate that the LEC vaccines against NP and M2e delivered with this platform provide complete protection from a lethal challenge 
(intranasal inoculation) with a $100 \times$ dose of influenza A/ H5N1/Vietnam/1203/04 whereas all naive mice succumbed to challenge (Shen et al., 2012). Therefore we conclude that surface EP DNA vaccination at the highly tolerable lowvoltage parameters can not only induce robust immune responses but also elicit responses that are sufficient for protection against lethal virus challenge. This is significant because the true test of any vaccine platform must be against a stringent challenge model.

The engineering and design of the SEP device are strongly tied to its future function as a clinical, prophylactic immunization device. Specifically, the electrode spacing on the surface device is $1.5 \mathrm{~mm}$ between pins. We believe this spacing to be crucial to the perceived tolerability of the device because it is directly related to the required field strength of the device. Indeed, we are currently researching the use of even shorter spacing with a view to essentially removing sensation from the procedure. Our SEP device also fires all 16 electrodes simultaneously with alternating rows of 4 electrodes applying reverse polarity (positive and negative). Simultaneous pulsing and the requirement for only three 100-msec pulses (with 100-msec delays) for optimal action mean that the entire EP treatment occurs in $0.5 \mathrm{sec}$. Perhaps most important for tolerability of the device, this device functions optimally with low-voltage parameters between 5 and $10 \mathrm{~V}$ ( 33 and $67 \mathrm{~V} / \mathrm{cm}$ ). The ability of the device to function at these low-voltage levels is partly due to the distinct electrode ground. The needle electrodes on this device are able to physically disrupt the stratum corneum layer of the skin, which is the primary barrier layer of this tissue, and make direct contact with the viable layers of the epidermis.

Our study indicated that applied electrical parameters between 5 and $10 \mathrm{~V}$ elicited sustained and robust humoral and cellular immune responses with no observable tissue damage. We believe that our SEP device, powered by these low-voltage parameters, represents an extremely mild EP platform rendering it an effective, highly tolerable, and patient-friendly approach to DNA vaccination. As such the SEP device might be suitable for delivery of prophylactic DNA vaccines that produce both antibody and cellular immunity in patients.

\section{Acknowledgments}

The authors thank Dr. Jonas Soderholm for advice on the linear construct design. The authors also thank Ms. Chris Knott for technical help on H\&E staining, and Mr. Jay B. McCoy and Mr. Steve Kemmerrer for support of the EP device. This work was supported in part by U.S. Army grant W23RYX-8141-N604: \#08023003

\section{Author Disclosure Statement}

The authors from Inovio Pharmaceuticals are employees of the company, and receive salary and stock options. Authors G.P.K. and A.B. have no actual or potential conflicts of interest.

\section{References}

Ahlen, G., Soderholm, J., Tjelle, T., et al. (2007). In vivo electroporation enhances the immunogenicity of hepatitis $C$ virus nonstructural 3/4A DNA by increased local DNA uptake, protein expression, inflammation, and infiltration of $\mathrm{CD}^{+} \mathrm{T}$ cells. J. Immunol. 179, 4741-4753.

Babiuk, S., Baca-Estrada, M., Babiuk, L.A., et al. (2000). Cutaneous vaccination: The skin as an immunologically active tissue and the challenge of antigen delivery. J. Control. Release 66, 199-214.

Babiuk, S., Baca-Estrada, M.E., Foldvari, M., et al. (2002). Electroporation improves the efficacy of DNA vaccines in large animals. Vaccine 20, 3399-3408.

Best, S.R., Peng, S., Juang, C.M., et al. (2009). Administration of HPV DNA vaccine via electroporation elicits the strongest $\mathrm{CD}^{+} \mathrm{T}$ cell immune responses compared with intramuscular injection and intradermal gene gun delivery. Vaccine 27, 54505459.

Bodles-Brakhop, A.M., and Draghia-Akli, R. (2008). DNA vaccination and gene therapy: Optimization and delivery for cancer therapy. Expert Rev. Vaccines 7, 1085-1101.

Bolhassani, A., Safaiyan, S., and Rafati, S. (2011). Improvement of different vaccine delivery systems for cancer therapy. Mol. Cancer 10, 3.

Broderick, K.E., Kardos, T., McCoy, J.R., et al. (2011a). Piezoelectric permeabilization of mammalian dermal tissue for in vivo DNA delivery leads to enhanced protein expression and increased immunogenicity. Hum. Vaccines 7, 22-28.

Broderick, K.E., Shen X, Soderholm J, et al. (2011b). Prototype development and preclinical immunogenicity analysis of a novel minimally invasive electroporation device. Gene Ther. 18, 258-265.

Brown, P.A., Khan, A.S., and Draghia-Akli, R. (2008). Delivery of DNA into skeletal muscle in large animals. Methods Mol. Biol. 423, 215-224.

Corr, M., Lee, D.J., Carson, D.A., and Tighe, H. (1996). Gene vaccination with naked plasmid DNA: Mechanism of CTL priming. J. Exp. Med. 184, 1555-1560.

Dobano, C., Widera, G., Rabussay, D., and Doolan, D.L. (2007). Enhancement of antibody and cellular immune responses to malaria DNA vaccines by in vivo electroporation. Vaccine 25, 6635-6645.

Donate, A., Coppola, D., Cruz, Y., and Heller, R. (2011). Evaluation of a novel non-penetrating electrode for use in DNA vaccination. PLoS One 6, e19181.

Draghia-Akli, R., Khan, A.S., Brown, P.A., et al. (2008). Parameters for DNA vaccination using adaptive constant-current electroporation in mouse and pig models. Vaccine 26, 52305237.

Ferber, D. (2001). Gene therapy: Safer and virus-free? Science $294,1638-1642$.

Fu, T.M., Ulmer, J.B., Caulfield, M.J., et al. (1997). Priming of cytotoxic T lymphocytes by DNA vaccines: Requirement for professional antigen presenting cells and evidence for antigen transfer from myocytes. Mol. Med. 3, 362-371.

Heller, L.C., Jaroszeski, M.J., Coppola, D., et al. (2007). Optimization of cutaneous electrically mediated plasmid DNA delivery using novel electrode. Gene Ther. 14, 275-280.

Heller, R., Cruz, Y., Heller, L.C., et al. (2010). Electrically mediated delivery of plasmid DNA to the skin, using a multielectrode array. Hum. Gene Ther. 21, 357-362.

Hirao, L.A., Wu, L., Khan, A.S., et al. (2008a). Combined effects of IL-12 and electroporation enhances the potency of DNA vaccination in macaques. Vaccine 26, 3112-3120.

Hirao, L.A., Wu, L., Khan, A.S., et al. (2008b). Intradermal/ subcutaneous immunization by electroporation improves plasmid vaccine delivery and potency in pigs and rhesus macaques. Vaccine 26, 440-448. 
Hirao, L.A., Draghia-Akli, R., Prigge, J.T., et al. (2010a). Multivalent smallpox DNA vaccine delivered by intradermal electroporation drives protective immunity in nonhuman primates against lethal monkeypox challenge. J. Infect. Dis. 203, 95-102.

Hirao, L.A., Wu, L., Satishchandran, A., et al. (2010b). Comparative analysis of immune responses induced by vaccination with SIV antigens by recombinant Ad5 vector or plasmid DNA in rhesus macaques. Mol. Ther. 18, 1568-1576.

Iwasaki, A., Stiernholm, B.J., Chan, A.K., et al. (1997). Enhanced CTL responses mediated by plasmid DNA immunogens encoding costimulatory molecules and cytokines. J. Immunol. 158, 4591-4601.

Jeon, S.H., Ben-Yedidia, T., and Arnon, R. (2002). Intranasal immunization with synthetic recombinant vaccine containing multiple epitopes of influenza virus. Vaccine 20, 2772-2780.

Kutzler, M.A., and Weiner, D.B. (2008). DNA vaccines: Ready for prime time? Nat. Rev. Genet. 9, 776-788.

Laddy, D.J., Yan, J., Kutzler, M., et al. (2008). Heterosubtypic protection against pathogenic human and avian influenza viruses via in vivo electroporation of synthetic consensus DNA antigens. PLoS One 3, e2517.

Laddy, D.J., Yan, J., Khan, A.S., et al. (2009). Electroporation of synthetic DNA antigens offers protection in nonhuman primates challenged with highly pathogenic avian influenza virus. J. Virol. 83, 4624-4630.

Lane, M.C., and Smith, W.C. (1999). The origins of primitive blood in Xenopus: Implications for axial patterning. Development 126, 423-434.

Lehrman, S. (1999). Virus treatment questioned after gene therapy death. Nature 401, 517-518.

Lin, F., Shen, X., McCoy, J.R., et al. (2011). A novel prototype device for electroporation-enhanced DNA vaccine delivery simultaneously to both skin and muscle. Vaccine 29, 67716780 .

Luckay, A., Sidhu, M.K., Kjeken, R., et al. (2007). Effect of plasmid DNA vaccine design and in vivo electroporation on the resulting vaccine-specific immune responses in rhesus macaques. J. Virol. 81, 5257-5269.

Luxembourg, A., Evans, C.F., and Hannaman, D. (2007). Electroporation-based DNA immunisation: Translation to the clinic. Expert Opin. Biol. Ther. 7, 1647-1664.

Maruyama, H., Ataka, K., Higuchi, N., et al. (2001). Skin-targeted gene transfer using in vivo electroporation. Gene Ther. 8, 18081812.

Mathiesen, I. (1999). Electropermeabilization of skeletal muscle enhances gene transfer in vivo. Gene Ther. 6, 508-514.

Mir, L.M., Bureau, M.F., Rangara, R., et al. (1998). Long-term, high level in vivo gene expression after electric pulse-mediated gene transfer into skeletal muscle. C. R. Acad. Sci. III 321, 893 899.

Muthumani, K., Lankaraman, K.M., Laddy, D.J., et al. (2008). Immunogenicity of novel consensus-based DNA vaccines against Chikungunya virus. Vaccine 26, 5128-5134.

Otten, G., Schaefer, M., Doe, B., et al. (2004). Enhancement of DNA vaccine potency in rhesus macaques by electroporation. Vaccine 22, 2489-2493.
Roos, A.K., Eriksson, F., Walters, D.C., et al. (2009). Optimization of skin electroporation in mice to increase tolerability of DNA vaccine delivery to patients. Mol. Ther. 17, 1637-1642.

Rosati, M., Bergamaschi, C., Valentin, A., et al. (2009). DNA vaccination in rhesus macaques induces potent immune responses and decreases acute and chronic viremia after SIVmac251 challenge. Proc. Natl. Acad. Sci. U.S.A. 106, 15831-15836.

Sardesai, N.Y., and Weiner, D.B. (2011). Electroporation delivery of DNA vaccines: Prospects for success. Curr. Opin. Immunol. 23, 1-9.

Shen, X., Söderholm, J., Lin, F., et al. (2012 Mar 7). Influenza A vaccine using linear expression cassettes delivered via electroporation. Vaccine [Epub ahead of print].

Tang, D.C., Devit, M., and Johnston, S.A. (1992). Genetic immunization is a simple method for eliciting an immune response. Nature 356, 152-154.

Tjelle, T.E., Rabussay, D., Ottensmeier, C., et al. (2008). Taking electroporation-based delivery of DNA vaccination into humans: A generic clinical protocol. Methods Mol. Biol. 423, 497-507.

Toebak, M.J., Gibbs, S., Bruynzeel, D.P., et al. (2009). Dendritic cells: Biology of the skin. Contact Dermatitis 60, 2-20.

Ulmer, J.B., Donnelly, J.J., Parker, S.E., et al. (1993). Heterologous protection against influenza by injection of DNA encoding a viral protein. Science 259, 1745-1749.

Wang, B., Ugen, K.E., Srikantan, V., et al. (1993). Gene inoculation generates immune responses against human immunodeficiency virus type 1. Proc. Natl. Acad. Sci. U.S.A. 90, 4156-4160.

Widera, G., Austin, M., Rabussay, D., et al. (2000). Increased DNA vaccine delivery and immunogenicity by electroporation in vivo. J. Immunol. 164, 4635-4640.

Wolf, A.I., Mozdzanowska, K., Williams, K.L., et al. (2011). Vaccination with M2e-based multiple antigenic peptides: Characterization of the B cell response and protection efficacy in inbred and outbred mice. PLoS One 6, e28445.

Zhang, L., Li, L., Hoffmann, G.A., and Hoffman, R.M. (1996). Depth-targeted efficient gene delivery and expression in the skin by pulsed electric fields: An approach to gene therapy of skin aging and other diseases. Biochem. Biophys. Res. Commun. 220, 633-636.

Zhang, L., Nolan, E., Kreitschitz, S., and Rabussay, D.P. (2002). Enhanced delivery of naked DNA to the skin by non-invasive in vivo electroporation. Biochim. Biophys. Acta 1572, 1-9.

Address correspondence to: Dr. Niranjan Y. Sardesai Inovio Pharmaceuticals 1787 Sentry Parkway West, Building 18, Suite 400 Blue Bell, PA 19422

E-mail: nsardesai@inovio.com

Receive for publication November 21, 2011;

accepted after revision May 9, 2012.

Published online: May 15, 2012. 